\title{
Sharing Background Noise: Enactive Approach in Reading Auditory Space
}

\section{SIGRADI2018 TECHNOPOLITICAS \\ xxii congresso da sociedade iberoamericana de gráfica digital 22th conference of the iberoamerican society of digital graphics 07|08|09|novembro|2018 iau usp | são carlos | sp br}

\author{
Altan Başık \\ Istanbul Technical University, Architectural Design Graduate Program | Turkey | \\ altanbasik@gmail.com \\ Sema Alaçam \\ Istanbul Technical University, Faculty of Architecture | Turkey | alacams@itu.edu.tr
}

\begin{abstract}
This paper conceptualizes the Auditory space in terms of hearing process by employing the Enactive Approach. In this context, this study aims investigate the spatial awareness and proposes a research methodology to achieve access to the auditory space where places share similar background noise. This methodology consists of two phases: field recording of the pre-determined route first explored by the Spectrogram Sound Analysis (SSA) technique, secondly with the participation of 8 subjects, a survey analysis based on listening to records captured from the predefined route. This research aims to reveal potential use of SSA by relating to survey examination as a new way of reading space.
\end{abstract}

Keywords Background Noise, Auditory Space, Enactive Approach, Spectrogram, Survey Examination

\section{INTRODUCTION}

Space can be defined as a geometrical form by the Euclidean Theory; however, this approach identifies only one feature of space which is experienced by the sense of sight. Space can be considered more complicated and multilayered cluster which consists of concurrent sensory interactions between the perceiving subject and the environment. Moreover, auditory space is one of the layers where this geometric form can still be perceived as an audible. As an example, humans are capable of recognizing the spatial depths and the texture of surfaces only by listening to the reflections of the voices in the space, if they trained for it. Nevertheless, auditory space does not only consist of the single audible voice but also noise. Neglecting the noise can be only possible in an anechoic chamber condition, where the absolute silence can be obtained. Apart from these, attention of the listeners play a crucial role on identification and characterization of the auditory space.

Each listener can pay attention to different voices and interpret them. Smalley (1996) explains the causes of this plural meaning production through the four listening mode by referring to the listening process concept of Pierre Schaeffer. The first mode is "an information-gathering" that listeners focus on the message of the sound.. Second is "passive reception" where voices become background noise unintentionally. The third and fourth listening modes are about musical listening. In the third listening mode, voices are abstracted by ignoring the messages of the voices intentionally and responding to attributes of sounds; as an example, listening to the traffic jam as a musical piece. Moreover, the fourth listening mode is also abstract listening the attributes of sounds although it is not necessary, it is a multimodal listening where four modes are implied in musical composition (Smalley, 1996).

In that manner, this study aims to understand a listening process in the context of the occurrence of the spatial awareness; therefore, we question how places, which share the same background noises, can be distinguished from each other just by listening. This question may become a part of many different research areas such as acoustic, cultural study, urban planning or architecture design. However, we approach this question as auditory space research in the field of architectural study and offer a new reading methodology for spatial explorations: at first, we conceptualize the framework of auditory space through the enactive approach to listening; and secondly, we examine the case study by criticizing the methodology of achieving access to the auditory space.

The proposed methodology of auditory space comprises of quantitative (environmental analysis) and qualitative (experiential examination) research by concerning the enactive approach. We employ the Spectrogram Sound Analysis (SSA) technique in the environmental analysis to illustrate the quantitative data of the soundscape recording. On the other hand, we focus on experiential analysis to unfold listeners' experiences through a survey study based on soundscape recordings.

The primary importance of this research, as our initial aim, is to reveal the correlation between quantitative and qualitative research. The reason of this attempt is derived from the enactive approach which claims that environment cannot be perceived as object or object, but coemergence of living-being and environment consist of each other. SSA type of data can be transformed 
simultaneously and interactively to different forms, for example 2D/3D visualization or spatial mechanics by taking advantages of current technology. These kinds of usage of SSA has a potential to create and design new experience for the users; however, SSA data does not provide directly any information about the experience of own audience Relatedly, sub-questions emerge: when the soundscape is visualized through SSA, can the analysis of the minor differentiations in the SSA graphics provide any meaningful information about the user experience? Is it possible to make any correlations between the analyzed visual data and the results of the survey? Taking these questions in mind, we aim to reveal the potentials of SSA as a way of reading space for the further design and research studies.

\section{CONCEPTUAL FRAMEWORK OF AUDITORY SPACE}

Auditory space might be understood as an audible sonic field which is determined by the limits of hearing under the effects of the acoustic property of the physical environment. Accordingly, auditory space is defined as a measurable field where the listening process does not occur yet. At this juncture, there needs to draw a simple distinction between hearing and listening; hearing can be defined as the passive receiving of a sound as input metaphor of receiving sound (Tomas\&Tuuri,2012), while the listening is an interpretation of voices which are selected by the listeners inside from the sonic environment. Accordingly, it can be concluded that when the hearing emerges in the body, the interpreted voices shape our spatial understanding and, thus, the limits of auditory field becomes abstracted and, thus, expanded by the listeners.

On the other hand, auditory space is also studied as a perception of a sound field regarding spatial hearing or sound localization (see also Carlile,1996). However, the question of how we perceive the world is broad and controversial study field to be concrete about auditory spatial perception. Therefore, this study conceptualizes the auditory space by taking into account the cognitive process of listening regarding the Enactive Approach and argues that auditory space cannot be indicated in physical measurements without denying the effects of the physical properties of the space on the spatial perception.

The fundamental understanding of the spatial experience is considered as it stars with being aware of self-location in the space. According to Groh (2014), the mental process of spatial awareness is a body-centred discovery that the whole body system work together in this process; moreover, the sense of hearing has an essential role on spatial awareness as much as the sense of sight and touch (for the effect of the vision on the spatial experience, see also Voss, 1996; Eimer 2004). She explains that the skills of an ear to distinguish the sound source location, and thus, the brain can find out the position of the body in the space. However, she also especially emphasizes that body-centered distances (spatial dimension) and the relationship between the body and the other locations be decoded in the brain by taking benefit from the cooperation of the sensory organs, the nervous system, the balance mechanism of the body and the memory.
From this point of view, it might be understood that our hearing skills are ready-being and we are so aware of auditory space. However, when we test our spatial awareness of hearing by closing our eyes and trying to walk around in the places that we do not know before, we may realize that finding the spatial clues is not easy only by listening. The reason for this situation is lack of practice to discover the spatial clues of the voices by listening; we may more use to practice of seeing. For example, blind individuals can have strong spatial awareness as much as the others who have no any disability. Visually disabled Daniel Kish explains that he improved his echolocation technique (making clicking noises with the mouth and listening reflection of sound from surfaces) by practicing, and thus, he better identifies the spatial depths, surfaces, and forms (Url-01, 2015).

It is not a new knowledge that blindness is not an obstacle for the formation of spatial awareness; they can play football or ride a bike. However, it can be questioned that what it looks like to be the sense of the hearing centered person, or, how the usage balance between hearing and seeing can influence our relationship with the environment? McLuhan (2014) discusses this issue, by referring to the article of Carothers(1959), between the cultures where the usage balance of the sense of sight and hearing have different domination. In this context, McLuhan explains the differences between non-illiterate native (who live more focused on the sense of hearing) and literate natives (who use the phonetic alphabet that establishes the domination of sense of sight), in that manner:

- non-literate natives live in the dynamic and multi-layered implicit meanings of sounds, and they see themselves as an insignificant part of a much larger organism;

- as a result of the invention of the Gutenberg Printing Press, the number of literate people who use the phonetic alphabet was increased. Thus, the need for voices was decreased the magical worlds of voices was reduced to the visual world's neutrality by the effects of the phonetic alphabet, and it developed the sense of sight centered culture which established the cause-effect relationship of worldview.(McLuhan, 2014)

In conclusion, McLuhan expresses that living in an aural or a visual world more focused has the potential to reshape the worldview; furthermore, he implicitly underlines the crucial role of the attention on perceiving the world. The attention can be considered as the creation of fore and background phenomenon and, thus, the next question can be that what is the role of the attention on listening process, or, more specifically, what is the background noise that we hear passively? Its known as cocktail party effect that the brain has an ability to selectively focus on particular voices by filtering them inside the hubbub; thus, we can shift our auditory attention one to the other voices. Besides that kind of fore and background creation, background noise is conceptualized as sonic unconscious by Cox (2009) to develop an ontology of sound.

Firstly, Cox approaches the notion of background noise as the ground of our perception by attributing to Serres's (1982) definition. He notes that no matter sounds can be 
heard or not, background noise is ceaseless and anonymous sonic flux that carries all the information of sounds. In this sense, this study considers the background noise as "information cloud" where all the possible meanings of interpreted sounds are hanging. Secondly, Cox consults Leibniz's (1704) "seashore" and "water mill" examples to define the unconsciousness concept by developing his proposal virtual and actual dimension of background noise. According to Leibniz, the person who walks on the seashore, his/her conscious cannot distinguish the particular noise of each wave of the sea. Besides, the person, who lives by the watermill can lose his/her listening conscious of the noises of the watermill in time, even though she/he gives full attention to it the beginning. With these examples, Leibniz especially underlies that these noises exist, no matter the listener's conscious is lost or not. His argument can be entirely related to Serres's conception of background noises which is ceaseless sonic as flux the ground of perception. Moreover, Leibniz's theory of "minute perceptions" defines the consciousness as an ability to capturing the particular noise of sea waves for a moment (Cox, 2009). In this sense, there can be concluded that background noise always exists as an information cloud and we hear the noises, at least for a moment, like having a section from this cloud. On the other hand, Cox criticizes Leibniz approach due to not considering technological advantages for the listening practices. Technological advances in recording and amplifying give an opportunity to listen to sound pieces in different places rather than the actual place where the sound event happens. As a result, the spatial information of actual sound becomes superposed with the new place where the actual sound is listening. Thus, this situation can lead emergence of plural meanings by the person who interprets the sounds.

Although it is attempted to infer from Cox's and Leibniz's approaches that how the space can be experienced within background noise, there still remains unclear questions about what is the conscious listening and how one perceives the space by listening. At this juncture, this research employs the enactive approach which is related to $4 \mathrm{E}$ cognitive theory. 4E cognition theory consists of Embodied, Embedded, Enacted and Extended mind theses, and the common ground of them is being against to the Cartesian cognitive theory (CCT). CCT claims that cognitive processes only occur in the brain (Rowland, 2010). Nevertheless, 4E cognitive theory is not totally disclaim the CCT, 4E theory strongly defend that cognition processes occur also out of the brain (Menary, 2010). The 'enactive' approach is developed under the title Embodied Mind (Varela, Thompson \& Rosch,1991) of the book and the celebrated discussions of experience, consciousness and related topics were studied allegiance to 'enactive' notions (Torrence, 2005).

This study mainly inherits Noe's (2004) conception of enactive perception which consists of sensorimotor knowledge (or knowledge of sensorimotor contingencies) and the ability to act on the world. However, Noe and his colleagues focused on vision even though they partially mention about hearing and tactile perception (O'reagan \& Noe, 2015). In relation with the active listening, Tuuri and Tuomas (2012) introduce hierarchical three modes from the bottom to the top: experiential, denotative, reflective. Tuuri and Tuomas (2012) consider the enactive approach within the experiential mode of listening, since the enactive listening is about acting through the sound as encounter for "knowing that" by exercising, probing and exploring before receiving the message (denotative) and abstracting (reflective) the meaning of the voices. On the other hand, Arteaga (2014) applies the enactive approach to auditory-architectural research, and he proposes the identification of four varieties of listening in order to achieve cognitive access to the environment: analytical, emotional, associative and imaginative listening. In this sense, Arteaga's listening modes differ from Tuuri \& Tuomas's (2012) approach on enactive listening. In Arteaga's interpretation of the enactive approach, the coemergence of living being and environment are taken into account. Environment can not be perceived as an object or object catalogue, but the co-emergence of living being and environment can produces the presences (Arteaga, 2014). Arteaga's approach carry similarities with Noe's conception of the phenomenological presence.

On this conceptual ground, Arteaga's proposal "four varieties of listening" can be analogized with to Cox's conception of background noise. The presences are in the continuous flux of production as same as the sonic flux of background noise. Moreover, capturing the presences from the moment through these listening modes resemble with having sections from the information cloud in a way of acting through the sound; these listening modes disclosure component of space as instant and subjective manner of presences. Therefore, those sections (four listening mode) can be considered an exact definition of the auditory space as the proposal of this research.

\section{THE CASE STUDY}

This study aims to employ Arteaga's "four varieties of listening" approach as an exploration of the auditory space experience (Arteaga, 2014). Arteaga discusses the framework of the four listening mode approach, however he does not give further details or a specific technique on demystifying the raw data into listening mode. In this study, we attempt to unfold each listening mode through applying a segmentation in the survey study. On the other hand, Arteaga's proposal focuses on only the listener's side of the concept that living being and environment coemerge. Thus, to be able to investigate the environmental part of this co-emergence, we employ qualitative research methodology; we propose the Spectrogram Sound Analysis (SSA) technique to explore the sonic environment which is recorded.

In this context, the case study is designed as an examination of the pre-determined route where the places share the same background noise. The case study consists of two phases:

At the first phase, a series of the field recordings were analyzed by the SSA technique; and as result of this analysis, obtained graphics of recordings are compared with each other to be able to reveal potential usage of SSA on the identification of the places of the route. The second phase consists of the survey examination in order to reveal subjects' experiences. The participants are expected to listen to the field recordings as the "living being" part of the co-emergence concept. 


\section{FIRST PHASE: INVESTIGATING THE AUDITORY FIELD OF THE ROUTE}

A series of audio recordings of a pre-determined route were collected in the first phase. The route covers different spatial characteristics located in an urban texture. These field records were taken by walking on the route of Atatürk streets in the neighborhood Heykel of Bursa city, Turkey. (Figure-1) The route starts on the one side of the Atatürk streets ("S" point) where trams and bus stations located. After the " $S$ " point, the route follows the underground passage between " $\mathrm{A}$ " and "B" point. From the " $A$ " point, the route goes down the staircase (S1) which is around $7 \mathrm{~m}$ in height; then it passes by the small shops of the underground passage. Before going out from the passage at " $\mathrm{B}$ " point, it came across with the few steps (S2) that go up. Between "B" and "C" point is the public square of the neighborhood. After "B" point, the route crosses the Pool $(P)$ where the water games are heard, then it goes down the few steps (S3) and arrives at the " $\mathrm{C}$ " point. At the " $\mathrm{C}$ " point, the route again goes down the stairs (S4) around $7 \mathrm{~m}$ and crosses "Branch Road" which is surrounded by high side walls. The route turns to the left at the "D" point, and it goes up to the "Back Street" with few steps(S5). Then after, it crosses "Side Street"(Ss) and the route is finished at the entrance of the old bazaar. On the starting point, the pedestrian way is divided in two part: one side passes under the columned buildings, and small shops are continuously placed in the entrance floor of it; the other side of the road is an open-air pedestrian way. This pedestrian way is connected to the other side of the street where the square locates with underpass passage. In this square opens to historical the Old Bazaar, Caravansary, Gazi Orhan Mosque and forecourt of Ulu Mosque. Thus this square is specialized as a transition and recreation area where peddlers serve snacks and drinks.

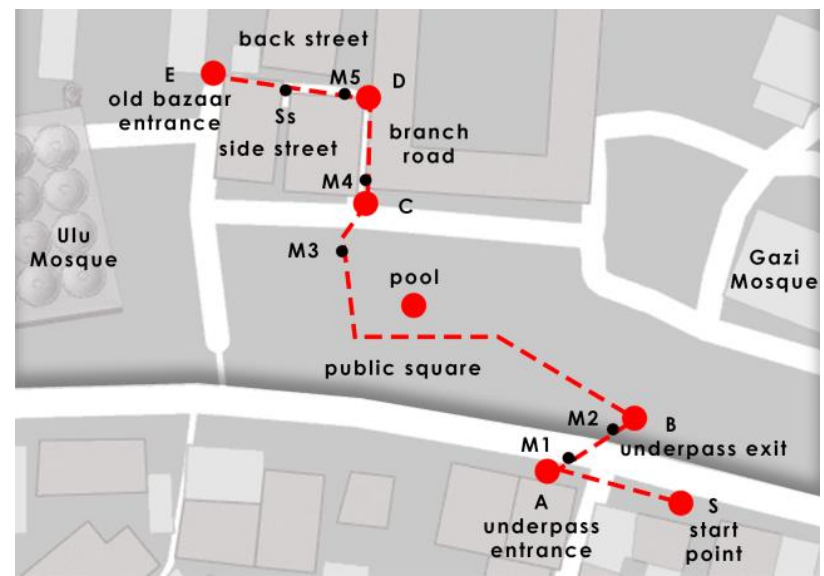

Figure 1: the route map. Source: authors

The first record was taken randomly on different days and times in order to explore everyday routines of place. During the process, night recordings could not be accomplished, since between the "C" and "D" points of the route was founded closed every day from 8 pm until 9 am and also the full day on Sundays because of the public security. On Saturdays and Sundays, the pedestrian traffic and sound event diversity were observed increasing through the day in the places of the route. Therefore, the case study is aimed to focus on Saturdays and records were taken at different times. Comparing, superposing and analyzing a series of record belonging the same route was helpful for capturing different aspects of the noise density differentiation and its relationship with the places located on the route. In other words, a manual spatial annotation was made based on the analysis of a series of records and their visual graphics provided by SSA.

Two different sound analysis methods were selected on the "E-analysis" interface, and as a result, two different graphics were obtained. The first graph was created by using the "rainbow" calculation mode (Figure-2). In the rainbow SSA graph, the different frequency ranges of voices are colored by regarding the sound volume range of voice, from high to low: red, yellow, green, blue and purple. This color distinction of frequency represents the relative location of the sound to the audio recording point. This colorization can be explained well as the "Doppler Effect" theory which explains when the same level of sound gets closer to listeners, it can be heard louder (Augoyard \& Torgue, 2005). Accordingly, red color frequencies are closer voices in regards to the microphone while the purples are distant. On the other hand, In the rainbow SSA graph, the specific level of density of the frequency range also inform the diversity of sound events at the moment. The density of purple colored frequencies ranges were seen high since the diversity of low volume sound event is varied. Therefore, purple colored frequencies are considered as background noise where the sound events are varied and far from recording point; moreover, the opposite is true for the red frequency range. The second graph is the sound density SSA graph, the frequency of the red color painted shows the overall loudness and the light blue color painted frequencies show the average amplitude level (Figure-3). As a result, these two graphics were helpful to represent the changes in sound intensities of the sonic environment of the route.

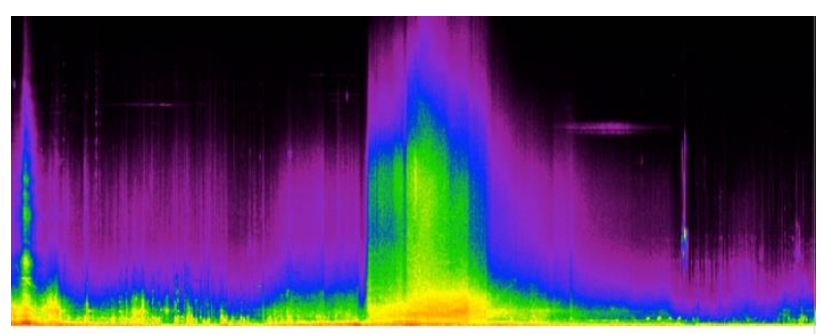

Figure 2: the rainbow spectrogram sound analyses. Source: authors.

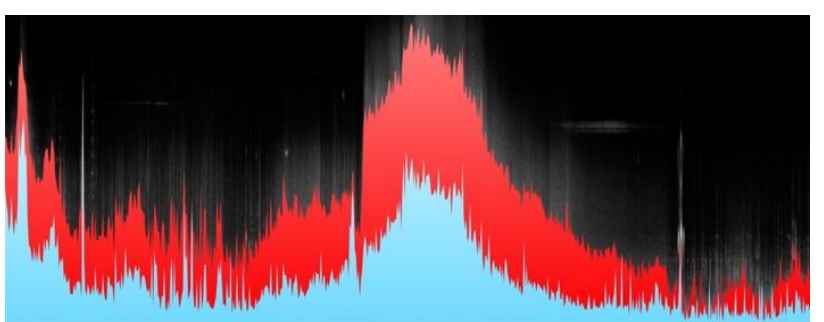

Figure 3: the sound density spectrogram sound analyses. Source: authors.

When the spectrogram graphs of the audio recordings are compared, some similarities and differences were 
observed (Figure-4). The reason for this situation in general:

- traffic noises can be heard almost in every place of the route, and thus, it maintains a steady level pink color frequency in every graph as background noise.

- the music from the shopping mall, the pedestrian traffic in the Underpass and at the public square and the noises of the water games inside the Pool are observed as transient sound events, and those do not be heard in every field recordings.
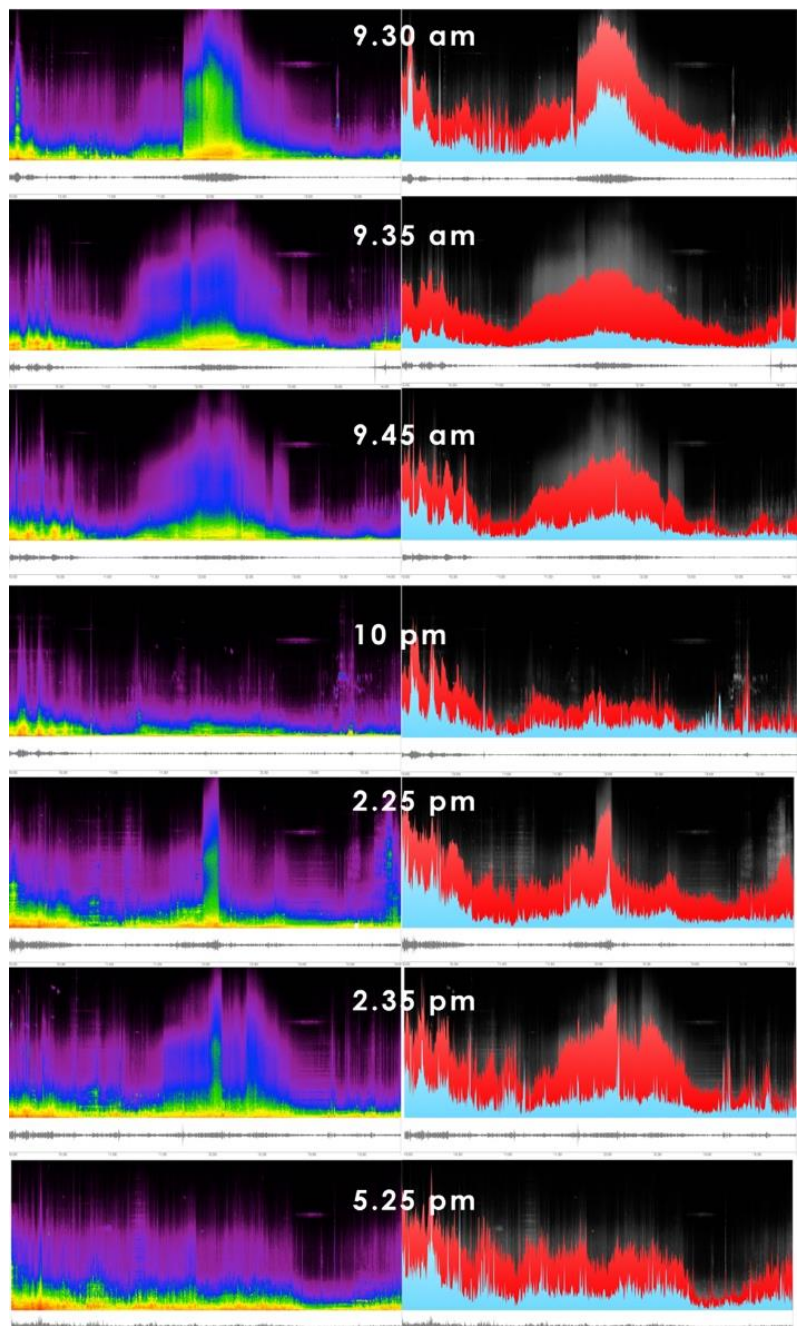

Figure 4: spectrogram analysis of the field recordings. Source: authors.

Further to the comparison process, the records taken at 9:45 am, 2:25 pm and 5:25 pm were selected in order to eliminate the similar graphs of audio recording. On the other hand, to be able to identify the places on the graph regarding the changes of the frequencies, the section of the route was adapted to spectrogram graphs. (Figure-5) after this application, these three graphs were compared. (Figure-6) accordingly:

- In the $5.25 \mathrm{pm}$ and $2.25 \mathrm{pm}$ SSA graphs, the background noise intensity level is found almost similar and high in contrast to the 9.45 am SSA graph, although some parts of 9.45 am SSA graph have the same level intensity of background noise with the others.

-The frequency range of the water game noises of the Pool is observed wide, and it can be only seen at 9.45 am and $2.25 \mathrm{pm}$ SSA graph in contrast to $5.25 \mathrm{pm}$ SSA graphs. Moreover, while getting closer to the pool and moving away from it, the frequency range of water game noises are getting increased and decreased.

- In the each SSA graph, the particular level of sound intensities decreases between "S" point and the end of the "S1" point, and also "C" and D point; on the other hand, before and after the "side street" the certain level increasing are seen.

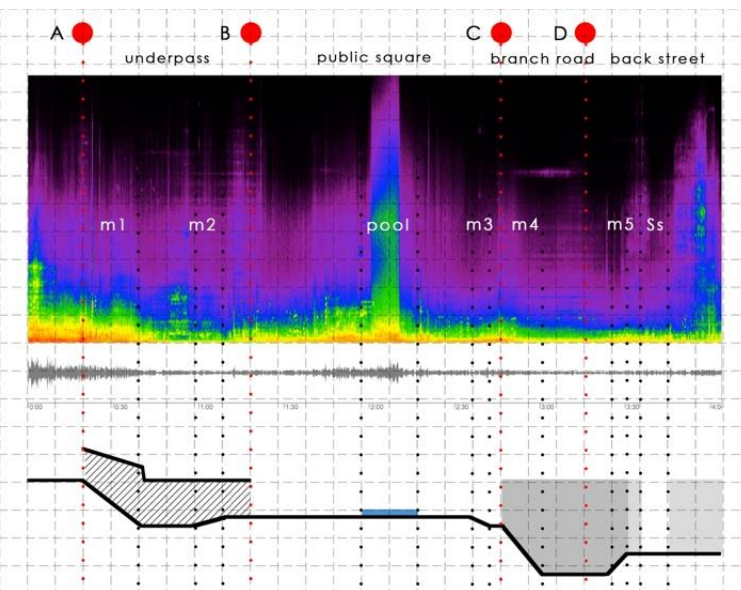

Figure 5: adaptation of the route section to 2.25 am the SSA graph. Source: authors.
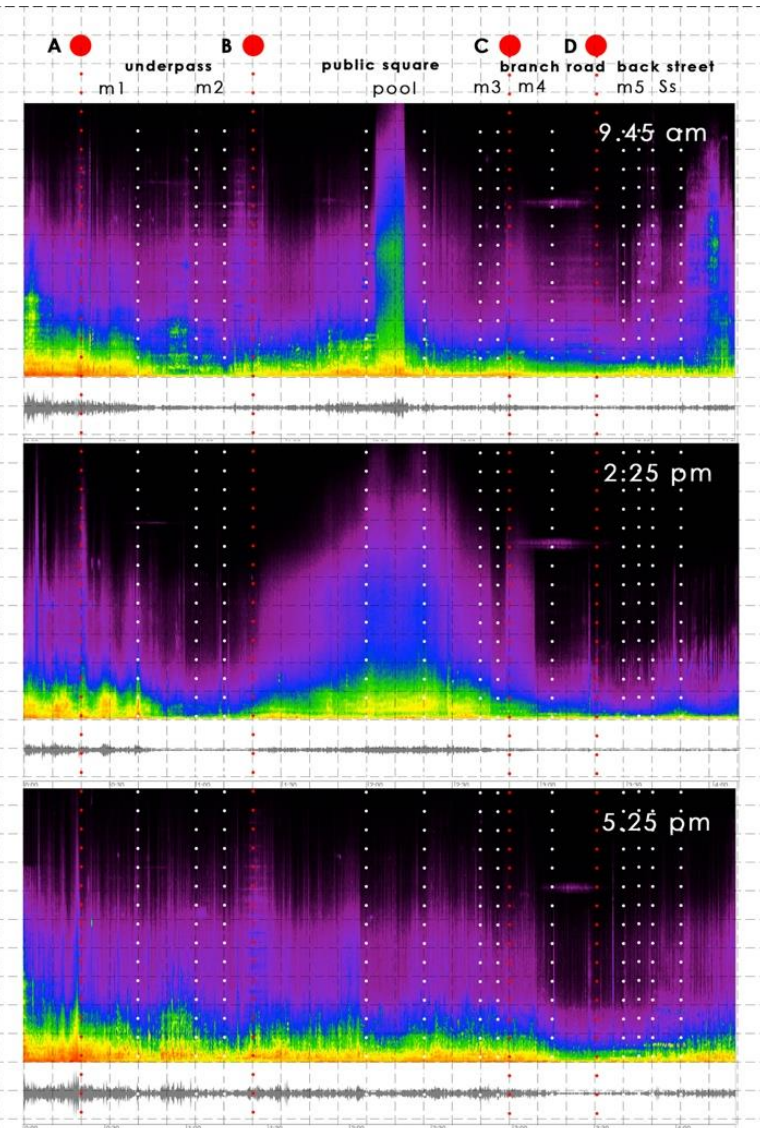

Figure 6: comparing the SSA graphs of the field recordings. Source: authors.

The reason for these changes on the graph can be explained as:

- The changes of sound events in the places: pedestrian and vehicle traffic noise densities low at 9.25 am, medium 
at $2.25 \mathrm{pm}$ and high at $5.25 \mathrm{pm}$ records; and between "C" and "D" points, any sound event does not occur.

- Mobility: recording the sonic environment of the route during walking, inherently, causes encountering the different auditory environments, even though some places become non-identifiable on the graph.

Apart from these, the acoustic character of the places might be considered as another reason; however, it cannot be examined through this comparison process.

As a result, we understood that to be able to distinguish the places from each other on the SSA graph, some specific sound density changes need to be present in the places. Nevertheless, this approach can not be applied in every situation when the same level of noise density dominates the whole route, such as 5.25 pm record. 2.25 $\mathrm{pm}$ record is considered as a generic record of the route since the characteristics sound events of the places can be heard without dominating by the intensive background noise. For example, in contrast to $2.25 \mathrm{pm}$ recording, vehicles and pedestrian traffic density are higher at 9.45 am recording and water noises of the Pool cannot be heard in $5.25 \mathrm{pm}$ record. Therefore, $2.25 \mathrm{pm}$ recording is selected to be used in the next phase examination.

\section{SECOND PHASE: SURVEY EXAMINATION}

The layout of the survey was developed from Arteaga's "four listening modes" approach: Analytic, Relational, Imaginative and Emotional. These four modes are concerned as the main sections of the survey. For each section, specific questionnaires are designed as a subsection, and the timeline of the field recordings is adapted to each subsection.

The Analytic Listening Mode (A-LM) section of the survey consists of three subsections in order to reveal the listener's awareness of "Spatial Typology", "Sound Density" and "Direction". (Figure-7) These three subsections are represented with different symbols on the timeline, and by doing so, subjects are enabled to answers the questionnaires by marking the symbols.

In the Spatial Typology (Ty) subsection, "open", "semiopen" and "closed" space typologies are needed to be unfolded. However, in contrast to closed space, distinct definition of the "open" and "semi-open" space typologies are not determined to the subjects in order to not to affect their experience. In the Sound Density(De) subsection changes of the low-medium-high level density of the audio recordings, and in the Direction(Di) subsection, the movements of the record (such as right, left, up, down, mobility and stability of) are questioned to be distinguished by the subjects.

Before starting the survey examination, the participants were encouraged to take notes concerning the Ty subjection during their listening. For example, when they mark the "open space" symbol somewhere on the timeline in the Ty section, they have to inform what is the sound density of this "open space" at the same marked point on the timeline in the Di subsection. Ty subjection is the first phase of the survey in where the participants start to describe their spatial awareness as abstract. Therefore, "open", "semi-open" and "closed" space typologies were introduced to the participants as pre-defined notations.

The way that how the participants develop their spatial typology awareness in the other section can be built in a structure, and as a result, it can be more readable. Nevertheless, this method is not a rule for the subjects; their unique approaches are also crucial for this research.

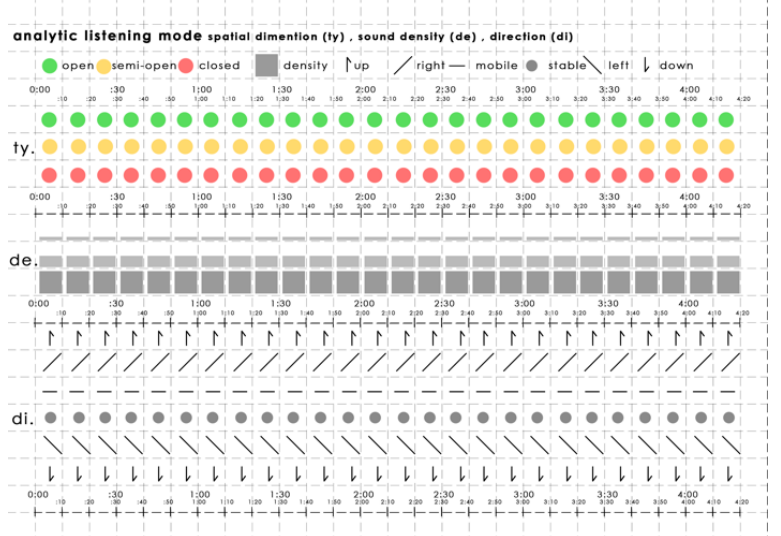

Figure 7: Analytic Listening Mode section of the survey. Source: authors.

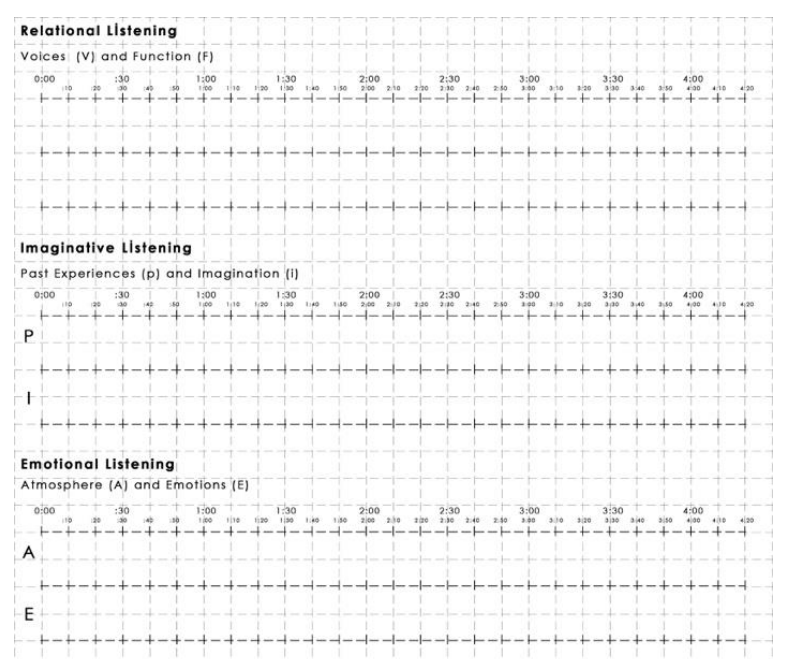

Figure 8: Relational, Imaginative, Emotional Listening Mode sections of the survey. Source: authors.

The last three sections (Figure-8) were the writing questionnaires in contrast to the A-LM section and aimed to reveal the subjects spatial imagination more concretely. In this sense, Relational Listening Mode (R-LM) section only focuses on the voices according to what the subjects specifically hear to be able to make the spatial distinction. Accordingly: in the "Voices"(V) subsection, subjects have to describe the voice regarding sound-causing events; and in the "Function"(F) subsection, they explain the spatial relation of these voices. For example, when the subject hears noises of cars, they can depict it as "it is a car or traffic jam noises" in the "V" subsection, and as "the cars are moving on the highway" in the F subsection. The Imaginative Listening Mode (I-LM) section concentrate on how the subjects envision the places of the voices what they indicated in R-LM sections according to their "Past Experiences" $(P)$ or non-experienced "Imagination" $(I)$, in the subsection "P" and "I". in the Emotional Listening Mode sections, subjects have to explain what are the Atmospheres $(A)$ of these places and what are their 
Emotional $(E)$ experiences of these atmospheres, in the sections $A$ and $E$. (Figure-8)

After subjects finished the survey examination, the survey answers, the SSA graphic and the route section were rearranged in order to obtain a readable outcome by comparing them in different combination. The critical part of these comparison tables is that spatial divisions are made visible on the timeline of the tables. During the comparison process, its seen that the answers of A-LM section can be readily brought together in the different tables, since A-LM section consists of symbolic representation; besides, the other sections could not be indicated in a symbolic order to reveal the semantic relation between them, they are kept as a personal information catalogue. As a result :

In the first graphic table (Figure-9), answers of Ty, De, Di subsections are grouped for each participant and listed together in order to read the personal experiences in the relationship between these three sections. Before the preparation of the table (Figure-9), it was considered that the table could have had a potential to reveal any specific pattern of personal experiences. As an example, whenever the high-level density is heard, it might be recognized as open-space typology by the subjects as the spatial experiential pattern of individuals or the group. However, the table (Figure -9) shows that patterns can be more complicated than an individual assumption, or there might be no pattern. At this point, the last three sections of the survey (A, I, E-LM) are employed to discover a clear understanding of the patterns. There has to be noted that this research concentrates on how the research methodology works rather than investigating all the possible patterns. Nevertheless, it's understood that this reading methodology has a potential of exposing the spatial experiences of each and it can be examined in further studies.

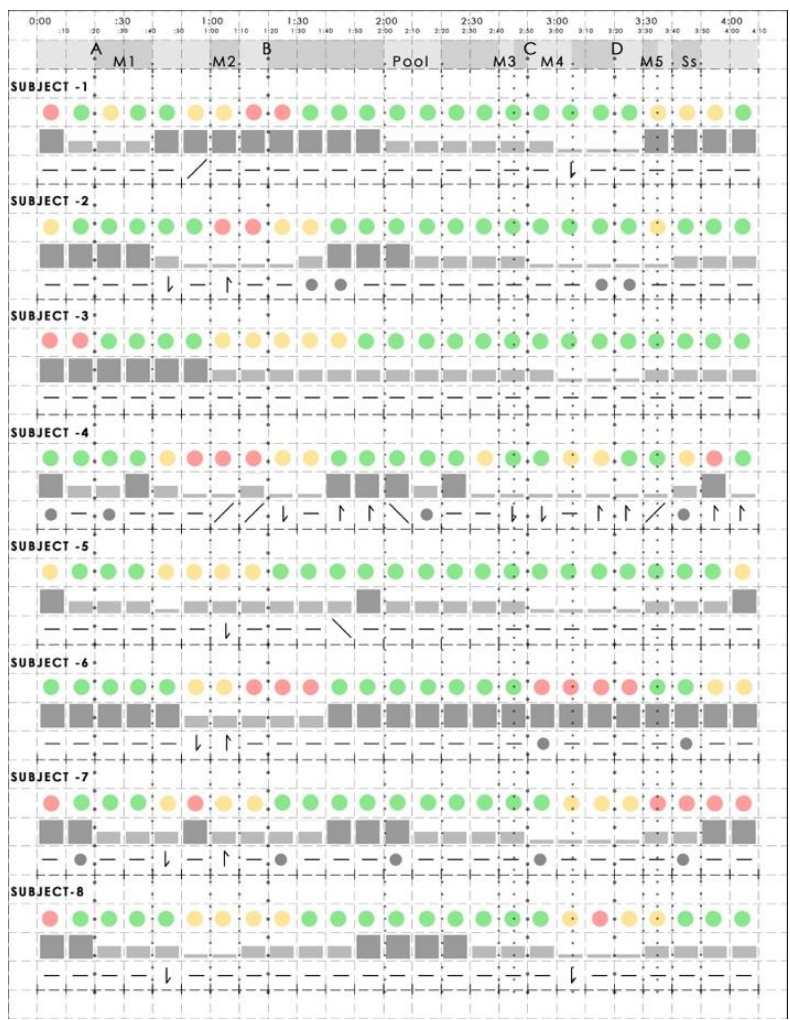

Figure 9: subject analyses. Source: authors.
In the following three graphic tables (Figure-10,11,12), Ty, De, Di subsections are designed as separate tables and the list of the subject group answers are included in each of them. In this regard, answers of the subjects are

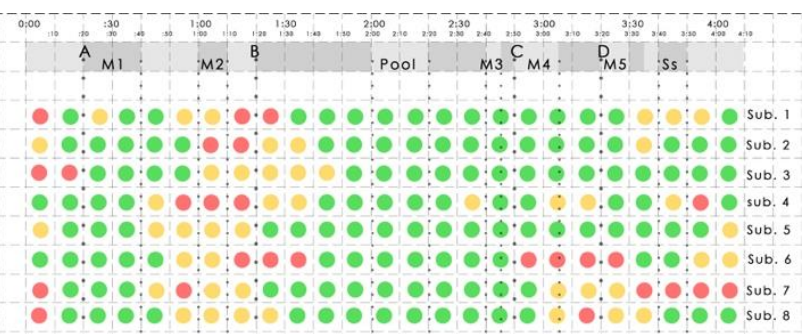

Figure 10: Spatial Typology (Ty) analyses. Source: authors.

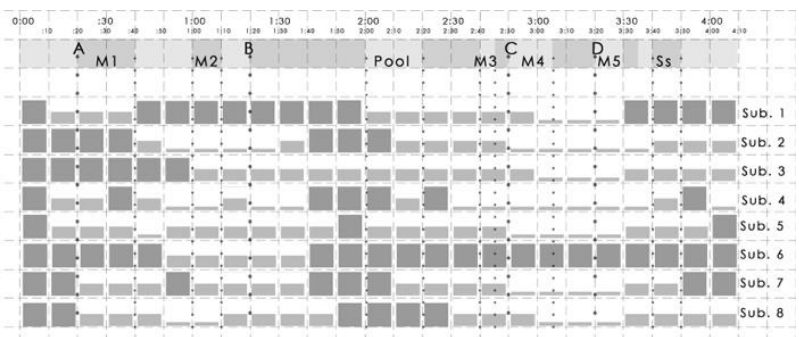

Figure 11: Sound Density (De) analyses. Source: authors.

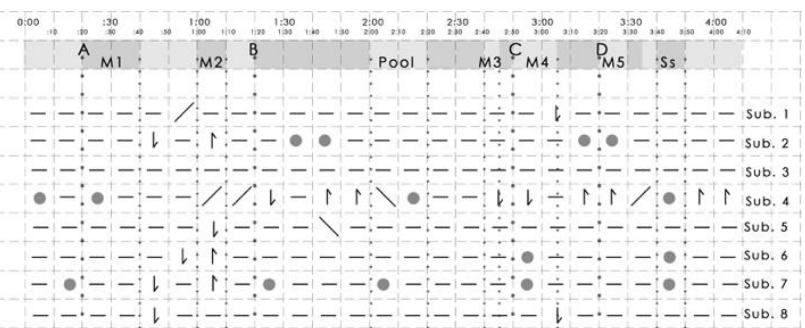

Figure 12: Direction (Di) analyses. Source: authors.

compared with each other, and thus, similar and conflict answers of the places are became visible. As example, in the Ty table, while the Pool is experienced as an openspace by each subject, its seen that subjects do not share the same experience on the Underpass (between the A and $\mathrm{B}$ point). Besides, in the De and the Di tables, there can not also be found the collective agreement about the "pool place" as same as the Ty table. these incompatible or similar answers for the same places in each tables considered an unexplainable or useless findings at the first sight. however, this tables show the crucial role of the scale on searching for meaningful outcome; as an example, there can be focused only one table by concerning subjects answer as group, or other way around, there can be also focused on only the answers of one person by concerning these three table.

In the last two tables (Figure-13,14), answers of the Ty and the De subsections are illustrated as a diagram form to be able to resemble with the SSA graphics. In the process of diagraming, the symbols of the De were flipped vertically due to corresponding with the graphics order of the Sound Density SSA graph. Besides, the section of the route is applied to the tables, and thus, spatial changes on the timeline become simultaneously readable with the SSA graph and the answer diagrams. As a result, while the Ty diagrams are compared Rainbow SSA graphic in order to understand the relationship between background 
noises and spatial typology (Figure 13), on the other hand, the De diagrams are related to the Sound Density SSA graphic to be able to seek the listening experiences of subjects (Figure 14).

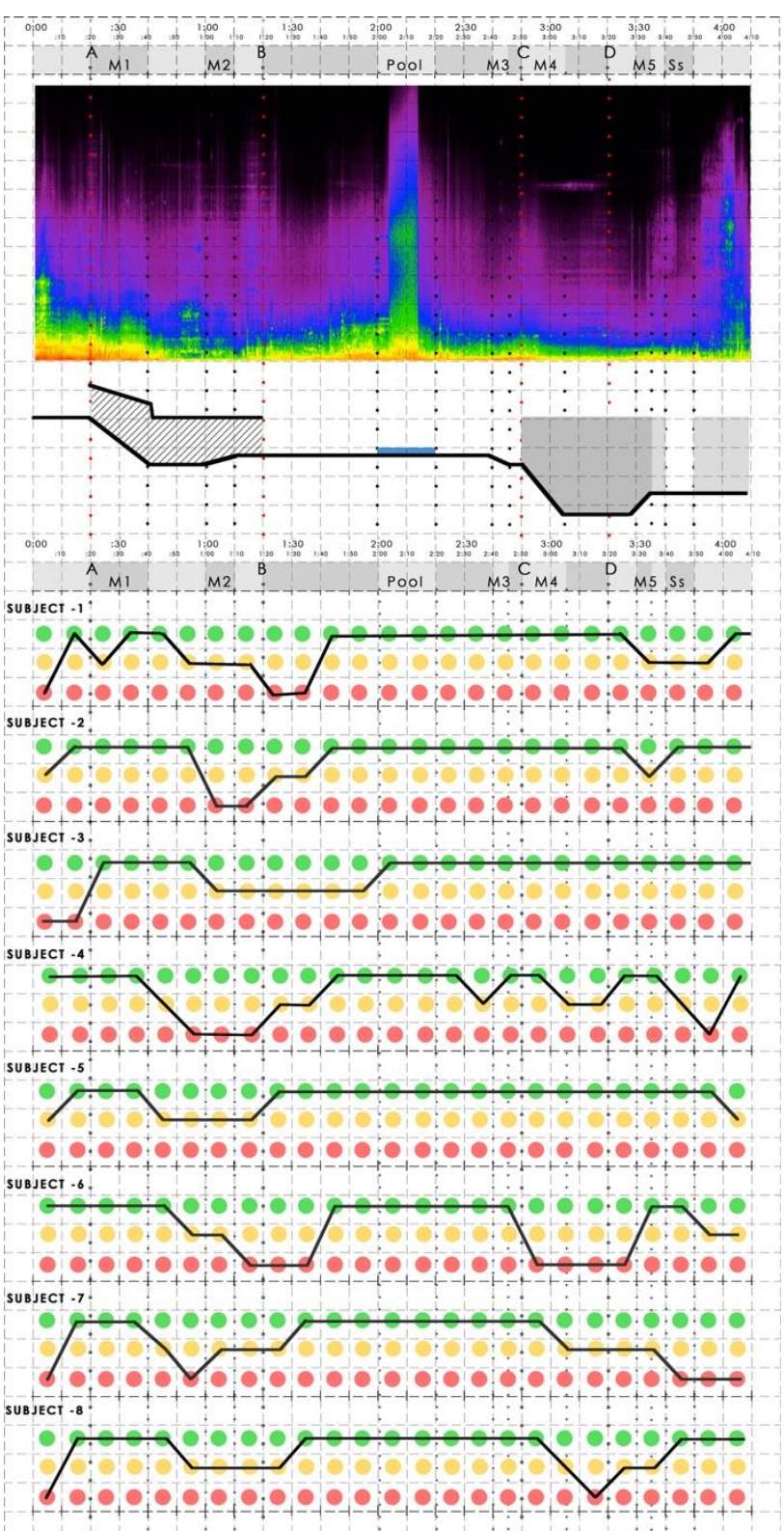

Figure 13: comparing Ty diagram of the subjects with Rainbow SSA graph. Source: authors.

According to these graphs, various similarities and differences are found in different combinations, and it can be interpreted as they are the precondition to each other. Therefore, results can be distinguished into two type information as spatial and behavioral. As an example for spatial examination of tables, in the Figure-10 and Figure13 tables where Ty answers of the subjects are compared as group, its seen that each subject contradictory marked the semi-open or the closed space symbols in between the beginning of $\mathrm{M} 2$ and $\mathrm{B}$ points. Accordingly, the subjects convinced about between these two points is not an open space. Furthermore, When it's looked on the SSA graphic in the Figure-13 for this place, it's seen that the specific level of noise density (purple colors) is protected; however, the other colors on this SSA graphic is in change before (decreasing) and after (increasing) that place (also see SSA graph the Figure-14).
Accordingly, there can be conclude that this place is recognized as non-open threshold space by each subject. On the other hand, when the personal information is wanted to be examined, there needs to be employed the the A-LM, I-LM and E-LM answers. As an example, when its looked at this threshold places again in the Figure 14, there can not be find similarities. However, according to A,I,E-LM answers, its understood subject took care of the sound events such as the pedestrian's noise, steps, kid's conversations and the toys' music, and these voices as reminds them that they are in certain type of inner space. Moreover, they specifically mentions that they are in different place at place since the traffic noises becomes reduced. As result, subjects are discovering spatial typology by taking advantages not only from the particular voices but also changes of background noises.

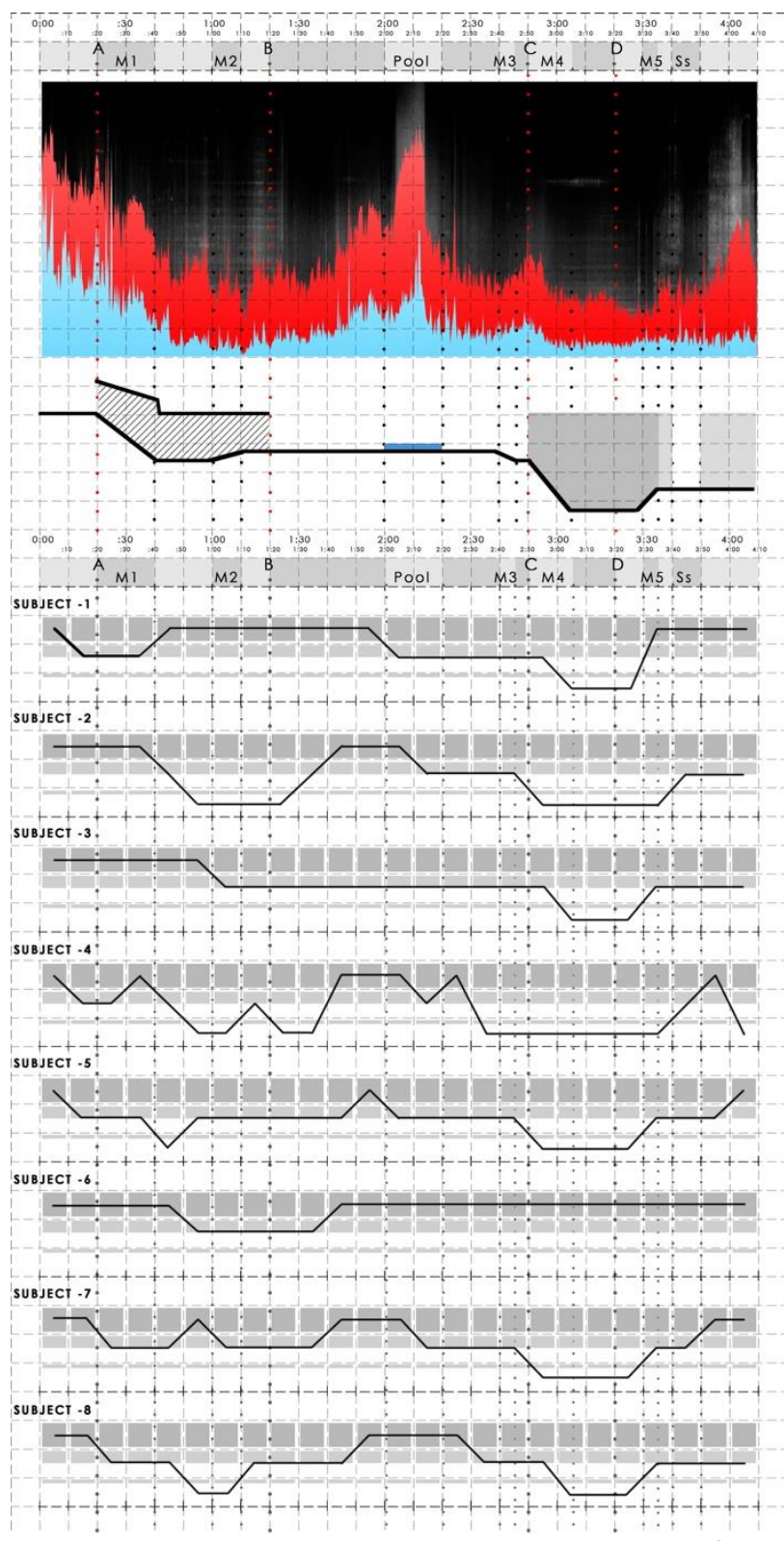

Figure 14: comparing De diagram of the subjects with Sound Density SSA graph. Source: authors. 


\section{CONCLUDING REMARKS AND DISCUSSION}

As a result, when we only consider SSA graphics usage to identify the places by seeking through the changes on the frequency range of the background noises, it's understood that it has own potential if only the places do not be dominated by the same sound densities. Therefore, we consider the SSA techniques as it works uniquely on the places. For example, in this study, places like underpass, the pool, and the branch road where changes of the sound densities provide an advantage to be able to recognize them on the SSA graph in contrast to the places like "S3" and "S5" where the sound density levels do not change according to before or after them. On the other hand, the survey examination is not a useful tool, if we try to explore the properties of actual spaces of the route. Each subject has a different interpretation way and understanding about what the sounds actually are and where the sounds specifically locate. As an example, when we look at the $X X$ table, we can see $x x$ point subjects are conceived about its open space; however, when we check their answers for "I-ML", we can see how they describe their open space estimation in real life are so different from each other.

Thus, it has to be noted that this study does not intend to explore the actual spatial properties of the places of the route by the survey examination and SSA technique. On the contrary, emerging contradictions between the reality and imagination (subjects listening experiences) are the desired findings of this research since they represent subjectivity of spatial experiences as the proof of enactive approach on environmental perception which consists of living being and environment co-emergence. Moreover, by comparing the SSA graphic with the survey examination and also with the section of the route in different combination, its found that causes of contradictions can be explained in regards to similarities. Therefore, the comparison tables (between the Figures-9 \& 14) are considered as a potential to unfold the multilayered presences of the auditory space and are the primary outcome of this research as a multiple reading approaches for auditory spaces.

As the further studies, the route can be changed, or it can be only focused only one place while this research can be specialized for just one person or different subject groups. By varying the research concept, SSA graphics and the Survey examination can result in different outcomes and interoperation of them unique in the place.

\section{REFERENCES}

Arteaga, Alex (2014), Thinking the Environment Aurally : An Enactive Approach to Auditory, Architectural Research and Design, Invisible Places Proceedings , Portugal.

Augoyard, Jean-François \& Torgue, Henry (2005), Sonic Experience : A Guide to Everyday Sounds, McGill-Queen's University Press

Carothers, J.C. (1959), "Culture, Psychiatry and the Written Word," in Psychiatry, Nov.

Cox, Cristoph. (2009), Sound Art and the Sonic Unconscious, Organised Sound 14(1):19-26, Cambridge University Press.

Eimer,Martin (2004), Multisensory Integration: How Visual Experiences Shapes Spatial Perception, Current Biology, Vol. 14, R115-R117, Elsevier Science Ltd.

Groh, M. Jennifer (2014), Making Space, The Belknap Press of Harvard University Press.

Menary, Richard (2010), Introduction to the Special Issue on 4E Cognition, Phenomenology and the Cognitive Sciences 9:459-463, Springer Science+Business Media.

McLuhan, Marshall (2011), The Gutenberg Galaxy: The Making of Typographic Man, University of Toronto Press.

Noe, Alva (2004), Action in Perception, MIT Press.

Noe, Alva (2012), Varieties of Presence, Harvard University Press.

Leibniz, G.W. 1704. New Essays on Human Understanding. Ed. and trans. Peter Remnant and Jonathan Bennett. Cambridge: Cambridge University Press, 1981.

O'Reagan, J.Kevin \& Noe,Alva (2001), Sensorimotor Account of Vision and Visual Conciousness, Behavioral And Brain Sciences 24,939-1031,Cambridge University Press.

Rowland, Mark (2010) The New Science Of The Mind, MIT Press.

Serres, Michel (1982), Genesis. Trans. Genevie ve James and James Nielson. Ann Arbor: University of Michigan.

Smalley, Denis (1996), The listening imagination: Listening in the Electroacoustic Era, Contemporary Music Review 13:2, 77-107, Harwood Academic Publishers GMbH

Varela, J. Francisco \& Thompson, Evan \& Rosch, Eleanor (1991), The Embodied Mind: Cognitive Science and Human Experience, MIT Press

Voss, Patrice (2016), Auditory Spatial Perception without Vision, Frontiers in Psychology 7:1960.

Url-01, Ted (2015, March 31), Daniel Kish: How I use sonar to navigate the world, YouTube, Retrieved from https://www.youtube.com/watch?v=uH0aihGWB8U
9 